Doug Geisler, Eva K. Grebel, and Dante Minniti, eds.

\title{
Evolution of Globular Clusters Formed in Mergers
}

\author{
François Schweizer \\ The Observatories of the Carnegie Institution of Washington, \\ 813 Santa Barbara Street, Pasadena, CA 91101-1292, USA
}

\begin{abstract}
Globular clusters formed in galactic mergers (e.g., The Antennae) can now be studied at different stages of their evolution. In young merger remnants (e.g., NGC 7252) these "second-generation" globulars appear by the hundreds as young halo clusters of roughly solar metallicity. While at first bluer and much more luminous than old metal-poor globulars, they become redder after $1-1.5 \mathrm{Gyr}$ and can then be observed as still overluminous red clusters of intermediate age in perturbed-looking $\mathrm{E}$ and $\mathrm{S} 0$ galaxies (e.g., NGC 1316, 1700, 3610). There is evidence from the color distributions, projected radial distributions, and perhaps also luminosity functions that these clusters eventually assume the properties of red metal-rich globulars observed in many giant ellipticals. Studies of globular clusters in ongoing mergers and young remnants suggest that second-generation globulars form from giant molecular clouds shocked by the rapid pressure increase in the merger-induced starburst. This pressure-induced formation lends credence to Cen's (2001) argument that the general pressure increase during cosmological reionization at $z \approx 7-$ 15 triggered the near-simultaneous formation of the universal population of first-generation metal-poor globulars observed in galaxies of all types.
\end{abstract}

\section{Introduction}

Major mergers of gas-rich spirals trigger bursts of intense star and cluster formation. They also form remnants that in many respects resemble elliptical galaxies. The present review concentrates on recent progress in our understanding of the evolution of globular clusters (GCs) formed during mergers and on the growing evidence that seems to link such globulars to the metal-rich, second-generation GCs observed in elliptical and S0 galaxies with bimodal cluster distributions.

The merging process is now well understood. When two disk galaxies with massive dark halos collide, they experience dynamical friction that leads to orbital decay and merging (Toomre \& Toomre 1972). The accompanying violent relaxation due to the fluctuating gravitational field redistributes the matter into a characteristic form relatively well approximated by a $r^{1 / 4}$ law. Modern simulations of mergers of gas-rich disks (e.g., Barnes \& Hernquist 1996; Barnes 1998, esp. Plate 4) describe in considerable detail the fate of the stars, cool gas, and gas heated to X-ray temperatures. The resulting model remnants share many properties with observed ellipticals, something that no other elliptical-formation scenario has yet achieved. Especially striking is the speed with which these merg- 
ers occur: From the first close approach of two disks to their coalescence takes only about $1 \frac{1 / 4}{-1} 1 / 2$ typical disk revolutions or $\sim 1 / 3 \mathrm{Gyr}$.

This speed poses a challenge when we study GCs formed during ancient mergers. Whereas the merger and cluster-formation processes are essentially linear in time, our ability to determine cluster ages is more nearly time logarithmic. Consider that when we look back in time, the eleven ongoing mergers and young remnants that make up Toomre's (1977) well-known sequence cover one full decade in age, ranging from $\sim 0.1$ Gyr to 1.0 Gyr. Therefore, we can study the cluster-formation process along this sequence in considerable detail. Progress is also being made in studies of GC systems in intermediate-age merger remnants, which I here define as 1 - 7 Gyr old remnants and which - thereforecover another $85 \%$ of an age decade [log Age(yr) $=9.0-9.85]$. But when we study cluster systems in old merger remnants that formed 7-14 Gyr ago, all details appear compressed into a mere $30 \%$ of one age decade, log Age $(y r) \approx$ $9.85-10.15$, even though this interval covers fully one half of the age of the Universe. This explains both our difficulties in understanding the details of early cluster formation and the resulting profusion of theories.

Logically, this review should begin with the formation and evolution of GCs in ongoing mergers, of which The Antennae are the prototype. Yet, this subject is already well covered by Whitmore's and Mengel's reviews elsewhere in this volume. Hence, I concentrate here on describing the evolution of GC systems in young, intermediate-age, and old merger remnants.

\section{Globular Clusters in Young Merger Remnants}

Two advantages of studying GCs in young (0.3-1 Gyr) merger remnants are that (1) dust obscuration is much less of a problem than in ongoing mergers and (2) most bright point-like sources are true globular clusters. The second fact follows from the clusters' measured half-light radii and ages. These ages typically exceed $100 \mathrm{Myr}$, or $\sim 25-50$ internal cluster-crossing times $t_{\mathrm{cr}}$, and thus indicate that such clusters are gravitationally bound. In contrast, most clusters in ongoing mergers like NGC 4038/39 and NGC 3256 are younger than $\sim 30 \mathrm{Myr}$ or $\sim 10 t_{\mathrm{cr}}$ and may eventually disperse. Therefore, the time lapse between the peak of cluster formation and the completion of a merger helps separate the wheat (i.e., the GCs) from the chaff.

Globular cluster systems have been studied with the Hubble Space Telescope (HST) in four young merger remnants so far: NGC 1275, 3597, 3921, and 7252 (for references, see Table 1). In each of these remnants, there are about $10^{-2}-10^{3}$ point-like sources that appear to be luminous young GCs. Age dating based on broad-band photometry shows that the majority of these globulars formed in a relatively short, 100-200 Myr time span during the merger. The young GCs appear strongly concentrated toward their host galaxies' centers, half of them typically lying within a central projected radius of $\sim 5 \mathrm{kpc}$. In NGC 3921 there are also, in addition to the 102 GCs, about 50 fuzzier objects which Schweizer et al. (1996) called "associations." These associations have colors ranging from relatively blue to quite red and may be in the process of dispersing. They may represent an earlier stage of the "faint fuzzies" seen by Brodie (this volume) and collaborators in three early-type galaxies. In NGC 3921 only three of the 50 


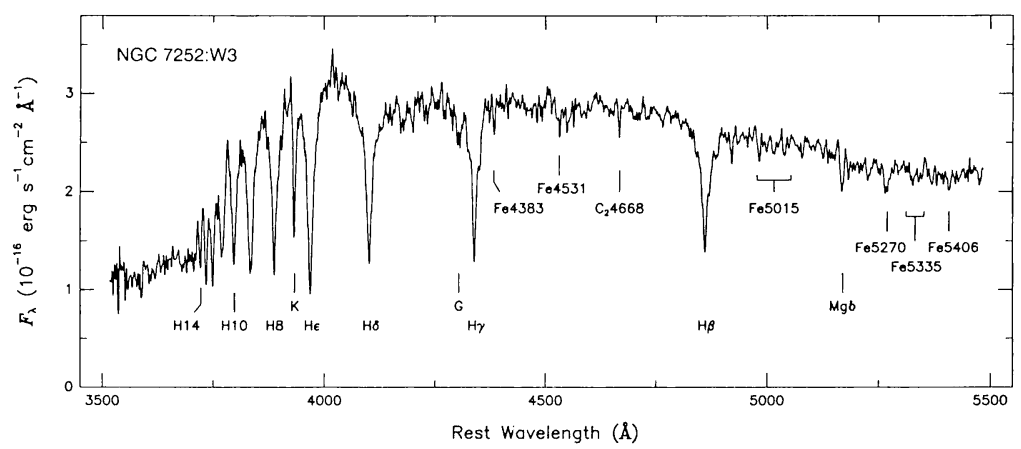

Figure 1. UV-to-visual spectrum of young halo cluster W3 in NGC 7252. Note strong Balmer absorption lines, the $\mathrm{K}$ line of $\mathrm{Ca}$ II, and relatively strong metal lines around $\mathrm{H} \beta$. This cluster has an age of 540 $\pm 30 \mathrm{Myr}$ and metallicity $[Z]=0.00 \pm 0.08$ (Schweizer \& Seitzer 1998).

associations lie within the central $5 \mathrm{kpc}$, presumably because most associations were too fragile to survive the intense churning at the merger's center.

The GC system of NGC 7252 has been studied in especially great detail. Spectroscopy of eight young GCs shows that seven of them feature strong Balmer absorption lines $[\mathrm{EW}(\mathrm{H} \beta)=6-13 \AA]$ indicative of a main-sequence turnoff dominated by A-type stars (Schweizer \& Seitzer 1998). Six of these GCs have ages in the narrow range $400-600 \mathrm{Myr}$. Figure 1 displays the spectrum of the brightest cluster, W3, with the Balmer and various metal lines marked. A first interpretation of this spectrum with Bruzual \& Charlot (1996) models for an assumed Salpeter IMF yielded a surprisingly high cluster mass of $1.8 \times 10^{8} M_{\odot}$. However, a reinterpretation of this spectrum with cluster models incorporating a new treatment of AGB stars yields a lower mass of $7 \times 10^{7} M_{\odot}$, or still $\sim 20$ times the mass of $\omega$ Cen (Maraston et al. 2001). These authors' $K$-band photometry indicates that most young globulars in the halo of NGC 7252 are presently in their AGB phase-transition stage (which lasts from 200 Myr to 1 Gyr).

The metallicities of these young halo GCs appear to be near solar. Figure 2a shows a $\mathrm{H} \beta-[\mathrm{MgFe}]$ diagram for the two globulars $\mathrm{W} 3$ and $\mathrm{W} 6$ (data points), from which $[Z]=0.00 \pm 0.08$ for $\mathrm{W} 3$ and $+0.10 \pm 0.17$ for W6. A fascinating object is S101, a very young halo cluster located in an $\mathrm{H}$ II region that is falling back into NGC 7252 from a tidal tail and has a metallicity of $[Z]=-0.12 \pm 0.05$. This cluster, located at $15 \mathrm{kpc}$ projected distance from the center, suggests that young globulars can form with considerable time delays when tidally ejected gas comes crashing back into a remnant.

The line-of-sight velocity dispersion of the eight spectroscopically observed GCs in NGC 7252 is $140 \pm 35 \mathrm{~km} \mathrm{~s}^{-1}$, leaving little doubt that these clusters belong to a halo population. From $H S T$ photometry, there appear to be a few hundred similar young halo GCs in addition to the old GCs that must have been part of the halo populations of the two input spirals (Miller et al. 1997). Figure 3 shows a color-magnitude diagram of all GCs beyond $2 \mathrm{kpc}$ projected distance from the center and the corresponding color distribution. Notice in this diagram the uniformly blue $(V-I)_{0}$ colors of the luminous young clusters, producing 

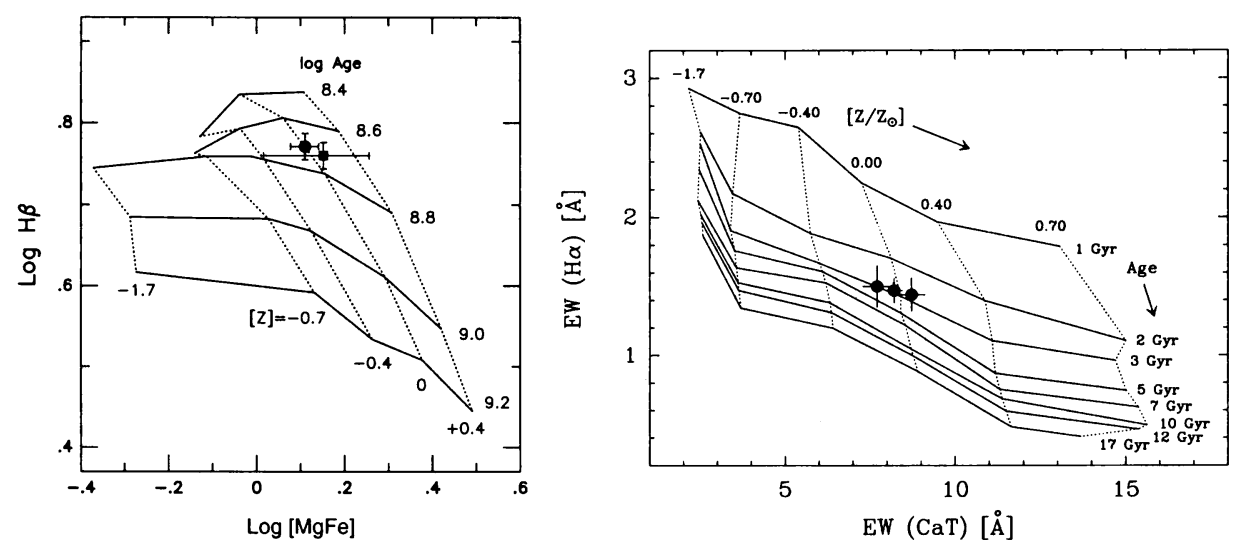

Figure 2. (a) (left) $\mathrm{H} \beta-[\mathrm{MgFe}]$ diagram for two GCs in NGC 7252 (Schweizer \& Seitzer 1998), and (b) (right) $\mathrm{H} \alpha$-Ca-triplet diagram for three GCs in NGC 1316 (Goudfrooij et al. 2001a). The NGC 7252 clusters are $\sim 550$ Myr old, the NGC 1316 clusters $\sim 3.0$ Gyr. Note that both sets of clusters have near-solar metallicities.

the pronounced narrow peak at $(V-I)_{0} \approx 0.65$ in the color distribution, and the parallel sequence of fainter, redder clusters, which shows up as a secondary peak around $(V-I)_{0} \approx 0.95$ in the color distribution.

In short, the merger of two gas-rich spirals in NGC 7252 has led to a young remnant with a clearly bimodal population of halo globular clusters. Besides the universal old metal-poor GCs the halo now also features a few hundred second-generation GCs that are young and metal-rich. As best as we can tell, the situation is similar in the young remnants NGC 3597 and NGC 3921, and perhaps also in NGC 1275. The many properties that remnants such as NGC 3597,3921 , and 7252 share with ellipticals suggest not only that these remnants are present-day protoellipticals (e.g., Schweizer 1998), but also that many E and S0 galaxies with bimodal cluster distributions may have formed their secondgeneration, metal-rich GCs in a similar fashion. Interestingly, the ratio of young to old GCs in NGC 7252 is about 0.7 (Miller et al. 1997), close to the mean ratio of metal-rich to metal-poor GCs observed in normal giant ellipticals.

\section{Globular Clusters in Intermediate-Age Merger Remnants}

If indeed E and S0 galaxies with bimodal cluster distributions formed through similar mergers as those described above, we should be able to (1) find E and S0 galaxies with second-generation GCs of intermediate age and (2) trace the evolution of second-generation GC systems from young through intermediate to old ages. Potential tracers of such evolution are, e.g., the GC color distributions, luminosity functions, and radial distributions.

Evidence for the existence of intermediate-age GCs in elliptical galaxies has been claimed for about eight systems, including NGC 1316, 5128, 1700, 3610, and 6702 (see Table 1 for refs.). The best case so far is NGC 1316 (=For A), for 

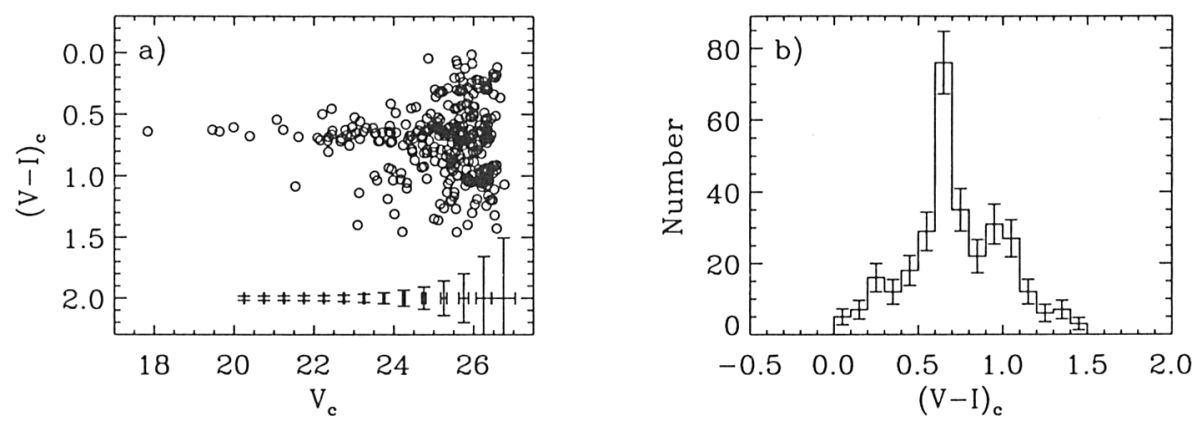

Figure 3. (a) $V-I$ vs. $V$ diagram for GCs in NGC 7252 and (b) corresponding color distribution. (From Miller et al. 1997.)

which there are spectroscopic data to bolster the claim made from broad-band photometry. Figure $2 \mathrm{~b}$ shows the equivalent widths of $\mathrm{H} \alpha$ for three bright GCs plotted versus the equivalent widths of their CaII triplets. From the superposed model grid, one can see immediately that all three GCs are about $3.0 \pm 0.5$ Gyr old and have close to solar abundances (Goudfrooij et al. 2001a). Their ages agree with the ages inferred from $B V I$ and $J H K$ photometry for a larger sample of GCs in this galaxy (Goudfrooij et al. 2001b; see also Goudfrooij, this volume). Therefore, the red peak of the bimodal color distribution for NGC 1316 clusters clearly contains GCs of intermediate age, and this merger remnant provides an evolutionary link between young remnants like NGC 7252 and old ellipticals with bimodal cluster distributions.

For the other galaxies with candidate intermediate-age GCs we have to rely on broad-band colors. Model simulations of bimodal GC populations with second-generation clusters of solar metallicity suggest what we might expect to observe at different ages (Whitmore et al. 1997, esp. Fig. 15): At 0.5 Gyr the second-generation GCs should appear both bluer and $\sim 2$ mag brighter than the old metal-poor GCs, as observed in the young remnants discussed above (e.g., Fig. 3). At 1.0-1.5 Gyr they should reach about the same $V-I$ color as old GCs, but still be $\sim 1.5$ mag brighter. At 3 Gyr they should be already distinctly redder than the old GCs but still 0.5-1 mag brighter, while at $\gtrsim 10$ Gyr they will appear both redder and slightly fainter. Figure 4 illustrates that this predicted crossover of GC colors indeed occurs. Shown are the color distributions of clusters in seven galaxies, with second-generation GCs ranging from very young and blue in The Antennae to old and red in M87. Whereas the general evolution from blue to red colors for second-generation GCs has been known for some time, the more detailed transition shown in the right-hand panels of Fig. 4 is new. The new data for NGC 1316 (Goudfrooij et al. 2001b), NGC 1700 (Brown et al. 2000), and NGC 3610 (Whitmore et al. 1997 and in prep.) diminish the gap in cluster ages from the previous $0.5-10^{+} \mathrm{Gyr}$ to currently about $0.5-3 \mathrm{Gyr}$.

The evolutionary trend as a function of age can be seen even better in the $\Delta(V-I)$ vs. $\Delta V_{10}$ diagram introduced by Whitmore et al. (1997). Figure 5 shows such a diagram, in which $\Delta(V-I)$, the reddening-corrected color difference between the peak due to second-generation GCs and that due to old metal- 


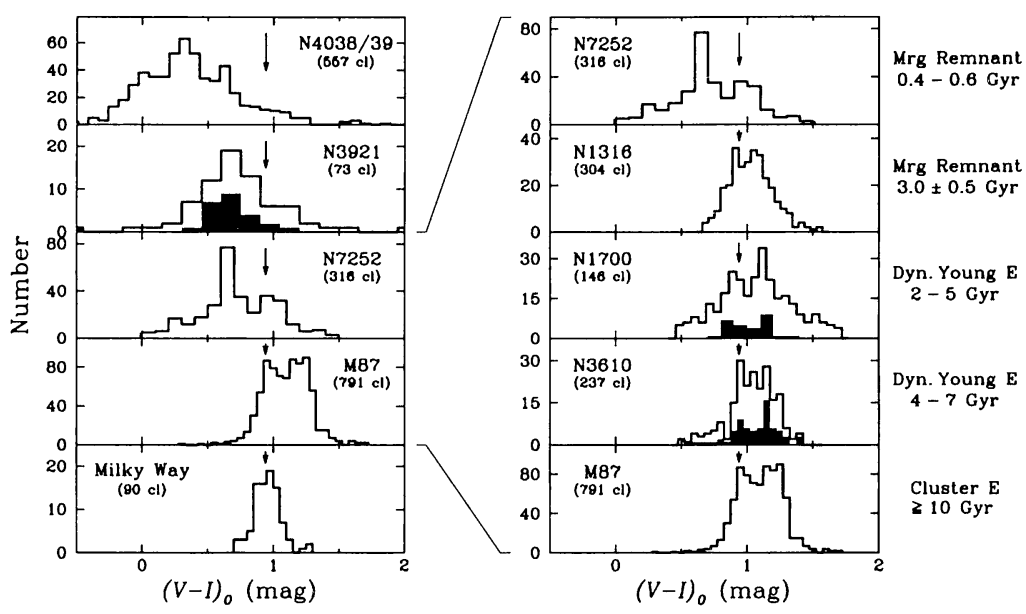

Figure 4. Color distributions of GCs in 3 mergers and 4 ellipticals, compared with Milky Way. Location of old metal-poor GCs is indicated by arrows. Note color evolution of 2 nd-generation GCs from very blue in N 4038/39 to red in M87. The five right panels show that the color crossover occurs between 0.5 Gyr and 3 Gyr (GC ages given in margin).

poor GCs, is plotted versus $\Delta V_{10}$, the magnitude difference between the 10thbrightest second-generation GC and the 10th-brightest old GC. Data points with error bars give the locations of the GC systems for the seven galaxies of Fig. 4. The data for NGC 1316 are new, as mentioned above. For NGC 1700, the old data point by Whitmore et al. (marked by a small cross) is superseded by new data from Brown et al. (2000), who succeeded in separating the secondgeneration GCs from the old GCs. Note that the GC systems lie roughly along the evolutionary track for solar-metallicity model clusters (solid line). This supports the notion that most second-generation globulars are relatively metalrich $([Z] \approx-0.8$ to +0.2$)$, as verified spectroscopically for GCs in NGC 7252, NGC 1316, and M87 (Cohen, Blakeslee, \& Ryzhov 1998). But above all, the $\Delta(V-I)-\Delta V_{10}$ diagram demonstrates quite clearly that the GC systems of the three merger galaxies and four ellipticals form an age sequence.

The luminosity function (LF) of second-generation GCs is another potential tracer of systemic evolution. The transition from the power-law form observed in young cluster systems to the log-normal form observed in old GC systems is now predicted theoretically (Fall, this volume). It is a consequence of the preferential erosion of low-mass clusters due to various disruption mechanisms, of which the main one is internal two-body relaxation and evaporation. This erosion should be evident in the observed LFs of second-generation GC systems that form an age sequence. So far, a tentative claim has been made only for NGC 1316 (Goudfrooij, this volume), where the slope of the LF of second-generation GCs appears to be shallower than the usual $\alpha \approx-1.6$ to -2.1 .

Finally, the radial distribution of GCs within their host galaxies contains valuable information about both their formation and their dynamical evolution. In the young remnants NGC 3921 and NGC 7252 the radial distribution of 


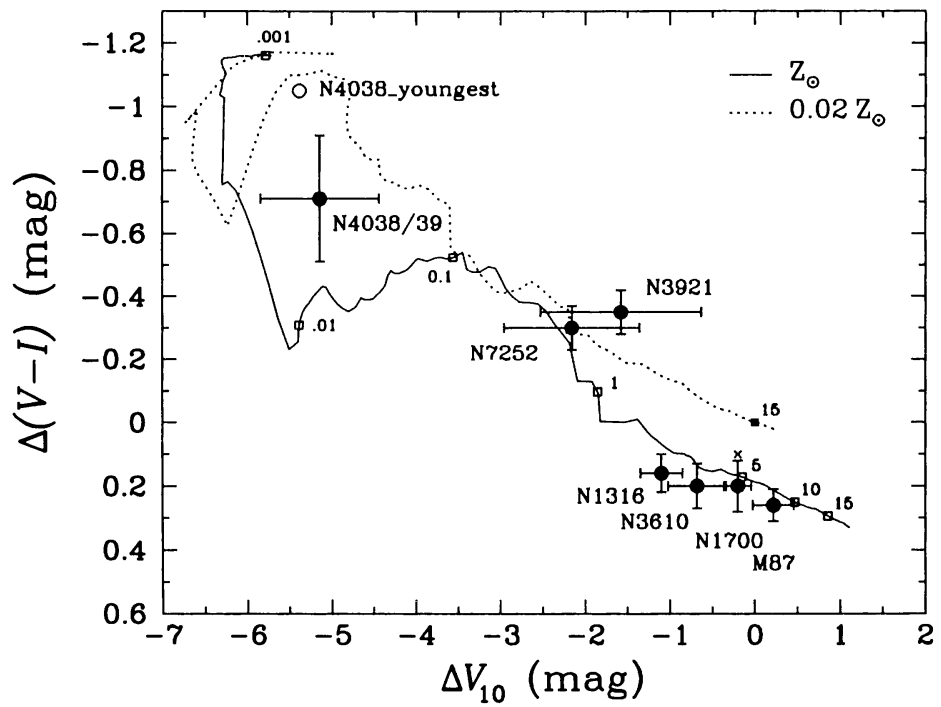

Figure 5. $\quad \Delta(V-I)-\Delta V_{10}$ diagram showing color difference between 2nd-generation and old GCs plotted vs. magnitude difference between 10th-brightest 2nd-generation GC and its old counterpart. Points with error bars show values for GC systems of 3 merger galaxies and 4 Es. Lines give evolutionary tracks for model clusters of solar and $1 / 50$ th solar metallicity (Bruzual \& Charlot 1996), and are marked with cluster ages in Gyr. Note that the observed seven GC systems form an age sequence. (After Whitmore et al. 1997, with some new data added.)

second-generation GCs is virtually identical to that of the galaxy light in $V$ (Schweizer et al. 1996; Miller et al. 1997). This indicates that the young GCs and their progenitors experienced the same violent relaxation as did the average star, suggesting that the GC progenitors were relatively compact giant molecular clouds orbiting among the disk stars of the two input spirals. There is tentative evidence for subsequent central erosion of GC systems, presumably due to tidal shocking of GCs during passages close to the center: At a radius of $1.2 \mathrm{kpc}$ in NGC 1316, the radial distribution of the GCs shows a deficit of $\sim 45 \%$ relative to the integrated star light (Goudfrooij et al. 2001b), while in old cluster ellipticals the corresponding deficit is significantly larger still (e.g., Capuzzo-Dolcetta \& Donnarumma 2001). Therefore, as with the color distributions, there appears to be a continuum of radial distributions of GCs ranging from young-cluster distributions as strongly centrally concentrated as the host merger remnants to old-cluster distributions typically less concentrated than the host ellipticals. It remains to be seen whether this tentative dynamical sequence will be confirmed as further examples of intermediate-age GC systems are added to the radialdistribution sample.

In short, second-generation globulars of intermediate age have recently been found in a few ellipticals and seem to form an evolutionary link between the young metal-rich GCs observed in recent merger remnants and the old metal- 

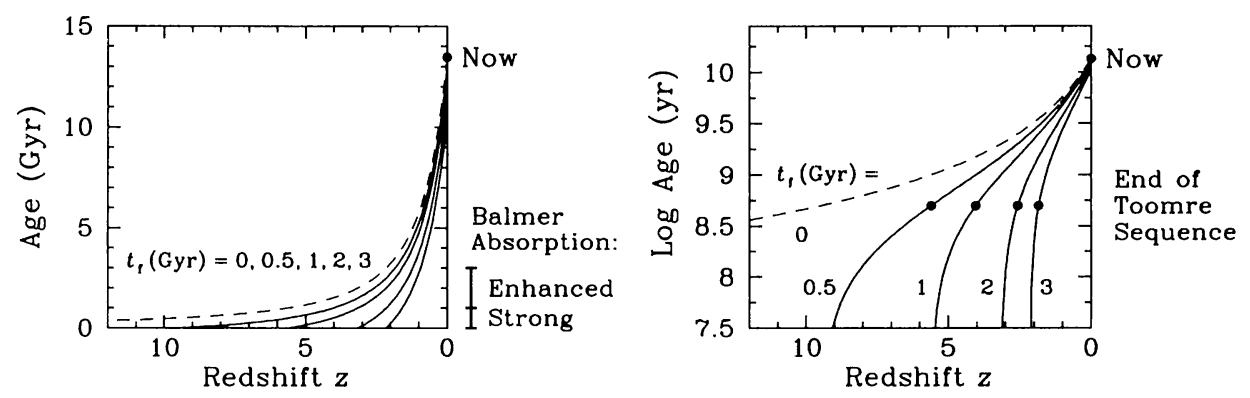

Figure 6. Linear ages (left) and logarithmic ages (right) of objects formed at epochs $0,0.5,1,2$, and 3 Gyr after Big Bang, plotted vs. redshift $z$ in a $\Lambda$ CDM cosmology $\left(H_{0}=70, \Omega_{\mathrm{m}}=0.3, \Omega_{\lambda}=0.7\right)$.

enriched GCs found in most old ellipticals. This link is seen most clearly in the color distributions of GCs, more tentatively in their radial distributions, and - for the moment-only weakly in their luminosity functions.

\section{Old Merger Remnants and GC Formation}

Given the propensity of globular clusters to form in the high-pressure environments of merger-induced starbursts (Schweizer 1987; Jog \& Solomon 1992; Ashman \& Zepf 1992; Elmegreen \& Efremov 1997), GCs in old merger remnants serve as valuable fossils of the galaxies' early star-formation history. The discovery of bimodal cluster populations in ellipticals (Zepf \& Ashman 1993; Whitmore et al. 1995) has convinced many sceptics that major mergers played a role in forming at least the metal-rich, second-generation GCs, though alternative formation scenarios have been proposed as well. A landmark paper was the observational study of NGC 4472 by Geisler, Lee, \& Kim (1996) who-based on photometry of thousands of GCs-discovered that the metal-rich clusters lie on average closer to the center than the metal-poor ones, as predicted by Ashman \& Zepf's merger model. This solved the old puzzle of why some GC systems show steeper radial abundance gradients than their host ellipticals, a fact attributable in NGC 4472 to the radially varying ratio of metal-rich to metal-poor GCs. Mergers involve much gaseous dissipation, which explains quite naturally the stronger central concentration of second-generation clusters.

Some recent attempts to age-date metal-poor and metal-rich GCs in cluster ellipticals have yielded comparable old ages of $>10 \mathrm{Gyr}$, albeit with error bars of several Gyr (e.g., Beasley et al. 2000; and Kissler-Patig, this volume). This begs two questions: (1) How well can we distinguish between "old" and "very old" ages? And (2), how much merger activity occurred during the period of uncertainty that corresponds to the large error bars?

Figure 6 attempts to address these questions. Linear and logarithmic ages of GCs formed at various epochs after the Big Bang are plotted as a function of redshift $z$ for the currently favored $\Lambda$ CDM cosmology. Note from the scale bars next to the linear-age plot that the enhanced Balmer absorption lines that facilitate spectroscopic dating during the first 3 Gyr fade by $z \approx 1$, even for 
GCs formed relatively late at $z_{\mathrm{f}} \approx 2$. Alternatively, the logarithmic-age plot illustrates that all GCs formed during the first $3 \mathrm{Gyr}\left(z_{\mathrm{f}} z_{2}\right)$ appear crunched into $\sim 0.1$ dex in age space from our vantage point ("Now"). Our current dating techniques for old stellar populations cannot resolve this tight age interval. Yet, given the rapidity of the merging process $(\sim 1 / 3 \mathrm{Gyr}$, see Sec. 1$)$, at least 10 major mergers could have occurred successively during the universe's first 3 Gyr. This illustrates that "comparable old ages" for metal-poor and metal-rich GCs do not in any way argue against the merger model of elliptical and metal-rich-GC formation. If metal-poor GCs formed very early on, metal-rich GCs may well have formed from a few subsequent disk mergers without us presently being able to measure the 1-3 Gyr age difference.

Perhaps the single most challenging question concerning GCs is why the old metal-poor GCs are so universally similar in all types of galaxies and environments. I believe that observations of GC formation in present-day mergers and starburst galaxies have yielded a crucial clue: Whenever an ensemble of giant molecular clouds is exposed to a rapid pressure increase, a significant fraction of these clouds get shocked and turn into GCs (Jog \& Solomon 1992; Fukui, this volume). The question then is: Was there a universal pressure increase early in the history of the universe that might explain the surprisingly uniform ages and properties of old metal-poor GCs?

Cen (2001) argues that the cosmological reionization at $z \approx 7-15$ provided just such a universal pressure increase, which in turn led to the synchronized formation of metal-poor GCs from early giant molecular clouds of low metallicity. If this hypothesis is correct, the following unified scenario of GC formation emerges.

Most globular clusters in the universe formed from shocked giant molecular clouds. The first-generation GCs formed near-simultaneously from pristine such clouds shocked by the strong pressure increase accompanying cosmological reionization. They populate all types of galaxies from dwarfs through spirals and ellipticals to giant cDs. Later-generation ("second-generation") GCs formed during subsequent mergers from metal-enriched giant molecular clouds present in the merging disks. Major mergers, some of which occur to the present time, led to elliptical remnants with a mixture of first- and second-generation GCs revealed by their bimodal color distributions. In relatively rare cases where such mergers occurred near cluster centers, disks stripped of their gas may have formed ellipticals with mainly first-generation GCs revealed by unimodal color distributions. Minor mergers tended to form S0 galaxies and early-type spirals, again with a mixture of first- and second-generation GCs. However, unlike in ellipticals many of the second-generation metal-rich GCs in disk galaxies may belong to a thick-disk population if they stem mainly from giant molecular clouds that belonged to the dominant input disk. Finally, a minority of "secondgeneration" GCs form sporadically from occasional pressure increases in calmer environments, such as in interacting Irregulars or barred galaxies.

Acknowledgments. I thank Bryan Miller for help with Figure 3, and him, Pat Seitzer, Paul Goudfrooij, and Brad Whitmore for their kind permission to reproduce figures. I also gratefully acknowledge research support from the NSF through Grant AST-99 00742 and from NASA through various HST grants. 
Table 1. GCs in Ongoing Mergers and Merger Remnants.

\begin{tabular}{|c|c|}
\hline Galaxy & References \\
\hline $\begin{array}{l}\text { NGC } 3256 \\
\text { NGC } 4038 / 39 \text { (Antennae) } \\
\text { NGC } 6052\end{array}$ & $\begin{array}{l}\quad \text { ONGOING MERGERS } \\
\text { Zepf+ } 99 \\
\text { Whitmore \& Schweizer 95; Whitmore+ } 99 \\
\text { Holtzman }+96\end{array}$ \\
\hline $\begin{array}{l}\text { NGC } 1275 \text { (Perseus A) } \\
\text { NGC } 3597 \\
\text { NGC } 3921 \\
\text { NGC } 7252 \text { (Atoms-for-Peace) }\end{array}$ & $\begin{array}{l}\text { YOUNG REMNANTS } \\
\text { Holtzman+92; Carlson+98; Spectra: Zepf+ 95; Brodie+ } 98 \\
\text { Lutz 91; Holtzman+96; Carlson+ 99; Forbes \& Hau } 00 \\
\text { Schweizer 96; Schweizer+96 } \\
\text { Schweizer 82; Whitmore+ 93; Miller+ 97; Maraston+ } 01 \text {; } \\
\text { Spectra: Schweizer \& Seitzer 93, } 98\end{array}$ \\
\hline $\begin{array}{l}\text { NGC } 1316 \text { (Fornax A) } \\
\text { NGC } 1700 \\
\text { NGC } 3610 \\
\text { NGC } 5018 \\
\text { NGC } 5128 \text { (Centaurus A) } \\
\text { NGC } 6702\end{array}$ & $\begin{array}{l}\text { NTERMEDIATE-AGE REMNANTS } \\
\text { Schweizer } 80 \text {; Shaya+ 96; Grillmair+ 99; Goudfrooij+ } 01 \mathrm{~b} ; \\
\text { Gómez+ 01; Spectra: Goudfrooij+ 01a } \\
\text { Whitmore+ 97; Brown+ } 00 \\
\text { Whitmore+ } 97 \\
\text { Hilker \& Kissler-Patig } 96 \\
\text { Graham \& Phillips } 80 ; \text { Harris }+84,92 ; \text { Zepf \& Ashman } 93 ; \\
\text { Minniti+96; Holland+99; Rejkuba 01; Spectra: Hesser+ } 84,86 \\
\text { Georgakakis+ }+01\end{array}$ \\
\hline
\end{tabular}

REFERENCES.-Brodie, J.P., et al. 1998, AJ, 116, 691; Brown, R.J.N., et al. 2000, MNRAS, 317, 406; Carlson, M.N., et al. 1998, AJ, 115, 1778; ditto 1999, AJ, 117, 1700; Forbes, D.A., \& Hau, G.K.T. 2000, MNRAS, 312, 703; Georgakakis, A.E., et al. 2001, MNRAS, in press; Gómez, M., et al. 2001, A\&A, in press; Goudfrooij, P., et al. 2001a, MNRAS, 322, 645; ditto 2001b, MNRAS, in press; Graham, J.A., \& Phillips, M.M. 1980, ApJ, 239, L97; Grillmair, C.J., et al. 1999, AJ, 117, 167; Harris, G.L.H., et al. 1984, ApJ, 287, 175; ditto 1992, AJ, 104, 613; Hesser, J.E., et al. 1984, ApJ, 276, 491; ditto 1986, ApJ, 303, L51; Hilker, M., \& Kissler-Patig, M. 1996, A\&A, 314, 357; Holland, S., et al. 1999, A\&A, 348, 418; Holtzman, J.A., et al. 1992, AJ, 103, 691; ditto 1996, AJ, 112, 416; Lutz, D. 1991, A\&A, 245, 31; Maraston, C., et al. 2001, A\&A, 370, 176; Miller, B.W., et al. 1997, AJ, 114, 2381; Minniti, D., et al. 1996, ApJ, 467, 221; Rejkuba, M., 2001, A\&A, 369, 812; Schweizer, F. 1980, ApJ, 237, 303; ditto 1982, ApJ, 252, 455; ditto 1996, AJ, 111, 109; Schweizer, F., et al. 1996, AJ', 112, 1839; Schweizer, F., \& Seitzer, P. 1993, ApJ, 417, L29; ditto 1998, AJ, 116, 2206; Shaya, E.J., et al. 1996, AJ, 111, 2212; Whitmore, B.C., et al. 1993, AJ, 106, 1354; ditto 1997, AJ, 114, 1797; ditto 1999, AJ, 118, 1551; Whitmore, B.C., \& Schweizer, F. 1995, AJ, 109, 960; Zepf, S.E., \& Ashman, K.M. 1993, MNRAS, 264, 611; Zepf, S.E., et al. 1995, ApJ, 445, L19; ditto 1999, AJ, $118,752$.

\section{References}

Ashman, K.M., \& Zepf, S.E. 1992, ApJ, 384, 50

Barnes, J.E. 1998, in Galaxies: Interactions and Induced Star Formation, ed. D. Friedli, L. Martinet, \& D. Pfenniger (Berlin: Springer), 275

Barnes, J.E., \& Hernquist, L. 1996, ApJ, 471, 115

Beasley, M.A., et al. 2000, MNRAS, 318, 1249

Brown, R.J.N., et al. 2000, MNRAS, 317, 406

Bruzual, A.G., \& Charlot, S. 1996, electronic tables of cluster models

Capuzzo-Dolcetta, R., \& Donnarumma, I. 2001, MNRAS, in press

Cen, R. 2001, ApJ, in press (astro-ph/0101197)

Cohen, J.G., Blakeslee, J.P., \& Ryzhov, A. 1998, ApJ, 496, 808

Elmegreen, B.G., \& Efremov, Y.N. 1997, ApJ, 480, 235

Geisler, D., Lee, M.G., \& Kim, E. 1996, AJ, 111, 1529

Goudfrooij, P., et al. 2001a, MNRAS, 322, 645 
Goudfrooij, P., et al. 2001b, MNRAS, in press

Jog, C.J., \& Solomon, P.M. 1992, ApJ, 387, 152

Maraston, C., et al. 2001, A\&A, 370, 171

Miller, B.W., Whitmore, B.C., Schweizer, F., \& Fall, S.M. 1997, AJ, 114, 2381

Schweizer, F. 1987, in Nearly Normal Galaxies, ed. S.M. Faber (New York: Springer), p. 18

Schweizer, F. 1998, in Galaxies: Interactions and Induced Star Formation, ed.

D. Friedli, L. Martinet, \& D. Pfenniger (Berlin: Springer), 105

Schweizer, F., Miller, B.W., Whitmore, B.C., \& Fall, S.M. 1996, AJ, 112, 1839

Schweizer, F., \& Seitzer, P. 1998, AJ, 116, 2206

Toomre, A., \& Toomre, J. 1972, ApJ, 178, 623

Toomre, A. 1977, in The Evolution of Galaxies and Stellar Populations, ed. B.M.

Tinsley \& R.B. Larson (New Haven: Yale Univ. Obs.), 418

Whitmore, B.C., et al. 1995, ApJ, 454, L73

Whitmore, B.C., Miller, B.W., Schweizer, F., \& Fall, S.M. 1997, AJ, 114, 1797

Whitmore, B.C., et al. 1999, AJ, 118, 1551

Zepf, S.E., \& Ashman, K.M. 1993, MNRAS, 264, 611

\section{Discussion}

P. Goudfrooij: One minor point for your consideration, on the metallicity of the GCs in NGC 7252: The gas collapsing to form the H II region of approximately solar metallicity in the outer region of NGC 7252 might have been enriched by, e.g., supernovae occurring when the massive, 300 - 500 Myr old GCs were formed.

F. Schweizer: This enrichment scenario seems unlikely. We know that the gas clump with the young cluster and H II region originated in the outer parts of one of the input spirals, since the farther out material lies in an interacting disk, the farther out it gets flung into the tidal tails and the longer it takes to fall back into the remnant. Therefore, the gas that formed the 400-600 Myr old GCs came from more central regions and should have been somewhat more metalrich than the currently returning gas clump, in agreement with the observations.

R. Kennicutt: In any picture where the first-generation clusters form by a universal process at very high redshifts (e.g., the Cen 2001 paper you cited), wouldn't you expect that the specific frequency and other properties of old blue clusters would be independent of galaxy properties? We have seen evidence earlier in this Symposium that the metal-poor halo clusters know about the environment in which they formed. Is this a problem for this picture?

F. Schweizer: I do not think that it is a problem. First, let me emphasize that in the Cen (2001) scenario only a small fraction of all giant molecular clouds get transformed into GCs, namely those with very low angular momentum. Cen concludes that the clumpy gas is reionized from outside in: As the first few generations of stars form, low-density regions are ionized first and higher-density regions later. Thus, there is more time for giant molecular clouds in denser 
regions to self-enrich before they get compressed and form GCs. I think this proposed sequence may have caused the metallicity gap that we are observing in the Milky Way halo, where the lowest-metallicity stars have $[\mathrm{Fe} / \mathrm{H}] \approx-4$ while the lowest-metallicity globulars have $[\mathrm{Fe} / \mathrm{H}] \approx-2.2$. In more massive galaxies, the lower abundance limit for GCs may be higher still.

D. Forbes: This is a comment. NGC 1052 shows plenty of signs of a recent merger, including $\mathrm{H}$ I tidal tails. Keck imaging reveals bimodality, but the red GCs are not young. So although we may have had a recent gaseous merger, few if any new globulars formed.

F. Schweizer: I know of the disturbed H I disk around NGC 1052, but not of any claims that NGC 1052 is a recent merger. From its $U B V$ colors, we estimated it to be a 7-9 Gyr old remnant (Schweizer \& Seitzer 1992, AJ, 104, 1039). 